\title{
Lethal variants in humans: lessons learned from a large molecular autopsy cohort
}

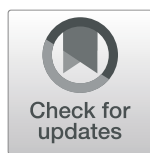

Hanan E. Shamseldin', Lama AlAbdi ${ }^{2}$ Sateesh Maddirevula ${ }^{1}$, Hessa S. Alsaif ${ }^{1,3}$, Fatema Alzahrani ${ }^{1}$, Nour Ewida$^{1}$, Mais Hashem', Firdous Abdulwahab', Omar Abuyousef ${ }^{1}$, Hiroyuki Kuwahara ${ }^{4}$, Xin Gao ${ }^{4}$, Molecular Autopsy Consortium and Fowzan S. Alkuraya ${ }^{1 *}$ (i)

\begin{abstract}
Background: Molecular autopsy refers to DNA-based identification of the cause of death. Despite recent attempts to broaden its scope, the term remains typically reserved to sudden unexplained death in young adults. In this study, we aim to showcase the utility of molecular autopsy in defining lethal variants in humans.

Methods: We describe our experience with a cohort of 481 cases in whom the cause of premature death was investigated using DNA from the index or relatives (molecular autopsy by proxy). Molecular autopsy tool was typically exome sequencing although some were investigated using targeted approaches in the earlier stages of the study; these include positional mapping, targeted gene sequencing, chromosomal microarray, and gene panels.

Results: The study includes 449 cases from consanguineous families and 141 lacked family history (simplex). The age range was embryos to 18 years. A likely causal variant (pathogenic/likely pathogenic) was identified in 63.8\% (307/481), a much higher yield compared to the general diagnostic yield (43\%) from the same population. The predominance of recessive lethal alleles allowed us to implement molecular autopsy by proxy in 55 couples, and the yield was similarly high (63.6\%). We also note the occurrence of biallelic lethal forms of typically non-lethal dominant disorders, sometimes representing a novel bona fide biallelic recessive disease trait. Forty-six disease genes with no OMIM phenotype were identified in the course of this study. The presented data support the candidacy of two other previously reported novel disease genes (FAAH2 and MSN). The focus on lethal phenotypes revealed many examples of interesting phenotypic expansion as well as remarkable variability in clinical presentation. Furthermore, important insights into population genetics and variant interpretation are highlighted based on the results.
\end{abstract}

Conclusions: Molecular autopsy, broadly defined, proved to be a helpful clinical approach that provides unique insights into lethal variants and the clinical annotation of the human genome.

Keywords: EHBP1L1, BMPR1A, MSN, FAAH2, Embryonic lethal, founder, loss of function, multi-locus

\footnotetext{
* Correspondence: falkuraya@kfshrc.edu.sa

${ }^{1}$ Department of Translational Genomics, Center for Genomic Medicine, King

Faisal Specialist Hospital and Research Center, Riyadh, Saudi Arabia

Full list of author information is available at the end of the article
}

(c) The Author(s). 2021 Open Access This article is licensed under a Creative Commons Attribution 4.0 International License, which permits use, sharing, adaptation, distribution and reproduction in any medium or format, as long as you give appropriate credit to the original author(s) and the source, provide a link to the Creative Commons licence, and indicate if changes were made. The images or other third party material in this article are included in the article's Creative Commons licence, unless indicated otherwise in a credit line to the material. If material is not included in the article's Creative Commons licence and your intended use is not permitted by statutory regulation or exceeds the permitted use, you will need to obtain permission directly from the copyright holder. To view a copy of this licence, visit http://creativecommons.org/licenses/by/4.0/ The Creative Commons Public Domain Dedication waiver (http://creativecommons.org/publicdomain/zero/1.0/) applies to the data made available in this article, unless otherwise stated in a credit line to the data. 


\section{Background}

Autopsy is intricately linked to medicine since antiquity and has been fundamental to our understanding of disease mechanisms. Unfortunately, however, there has been a steady decline in autopsy rates due to a combination of factors [1]. Leveraging the power of DNA to reveal the cause of death, Ackerman and colleagues coined the term "molecular autopsy" in 1999 when describing their approach of sequencing KVLQT1 in a cohort of young adults who died of unexplained drowning [2]. Since then, there has been a sustained growth of the literature on molecular autopsy with evolution of the technology used from targeted variant analysis to Sanger sequencing of one or more genes of interest to nextgeneration sequencing gene panels to whole-exome sequencing. However, the term remained largely confined to the study of unexplained death among young adults, e.g., a very recent "genomic autopsy" study reported the use of whole-genome sequencing to determine the cause of death in young adults averaging 23 years of age [3]. In 2018, we suggested an expansion of molecular autopsy to also include its application in maternal-fetal medicine (lethal malformation, stillbirths, intrauterine fetal deaths, etc.), which was subsequently endorsed by others $[4,5]$.

By focusing on the identification of lethal variants, molecular autopsy has the potential to make important clinical and scientific contributions to the field of human genomics. Defining the cause of death at the molecular level provides diagnostic precision, allows accurate genetic counseling, and enables management changes among surviving relatives. Furthermore, molecular autopsy improves the interpretation of the clinical sequencing of the human genome (gene-level and variant-level) by revealing "gene essentiality" [6] and helps expand the spectrum of phenotypic expression of genes. In addition, novel evolutionary insights can be gained by comparing the pattern of lethal variants in humans vs. model organisms [7]. Improved knowledge about lethal variants is also directly relevant to population genomics [8]. To maximize these benefits, we have sought in this study to utilize molecular autopsy broadly defined in a large cohort.

\section{Methods}

\section{Human subjects}

We used a broad case definition of any death before age 18 years with a suspected genetic etiology. The result of the molecular investigation, whether negative, ambiguous, or positive, will have been obtained after death. EDTA blood was obtained from 481 Saudi families (collected during the period 2009 through 2021), and in some instances, we also obtained sodium heparin blood or skin biopsy as needed. Samples were collected only after signing a written informed consent form by the parents. The research was approved by the local IRB (KFSHRC RAC\# 2080006, 2121053, 2140016, 2080033, and 2070023). The molecular tools used for the analysis of the families were exome sequencing $(n=383)$, gene panel $(n=80)$, positional mapping and targeted sequencing $(n=12)$, RNA-seq $(n=1)$, WGS $(n=1)$, and chromosomal microarray sequencing for all simplex cases. This heterogeneous testing modality was driven by the availability of certain genetic testing at the time of investigations. The study included 163 previously unpublished families.

\section{Variant identification and classification}

All cases recruited after 2012 were analyzed using exome sequencing (ES). Earlier cases were analyzed using targeted panels relevant to the clinical phenotype as described before [9]; negative cases were then submitted for ES. In a very small number of cases, positional mapping and targeted gene sequencing strategy $(n=12)$, RNA-seq $(n=1)$, or whole-genome sequencing $(n=1)$ were used. Candidate variants were selected on the basis of clinical relevance and were classified according to ACMG guidelines [10]. Cases were considered "solved" if a pathogenic or likely pathogenic variant was identified that explains the phenotype, ambiguous outcome refers to variants of uncertain significance (VUS, including variants in novel candidate genes); otherwise, the case was considered "negative." When DNA from the index is not available, duo ES was pursued in both parents (molecular autopsy by proxy) as described [4]. Chromosomal microarray (CMA) was applied on all simplex cases before subsequent analysis with ES or gene panels was pursued. Local minor allele frequency (MAF) was calculated based on an in-house dataset of 2379 local, ethnically matched exomes.

\section{Statistical testing}

$p$ values for statistical analysis of diagnostic yield were obtained using the chi-square test.

\section{Web resources}

The following web resources were used to document different relevant information as follows: Data from Deciphering the Mechanisms of Developmental Disorders (https:/dmdd.org.uk), a program funded by the Wellcome Trust with support from the Francis Crick Institute, is licensed under a Creative Commons Attribution license and was used for the phenotype of Ehbp111 knockout mice. Genome Aggregation Database https:// gnomad.broadinstitute.org [11] was used to check the frequency of identified variants in different populations. Pfam database, http://pfam.xfam.org/ [12], was used to determine protein domains and map the mutated base. Finally, ClinGen, https://clinicalgenome.org [13], criteria 
were applied to upgrade the classification of some variants by upgrading the gene-disease relationship.

\section{Results}

We enrolled 481 index cases in this analysis including 163 previously unpublished cases (Additional file 1: Table S1). The study cases represent a very wide range of phenotypes (see Additional file 2: Fig S1-S9 for representative images) and age at death: intrauterine fetal death (IUFD) $(n=50)$, pregnancy terminations due to lethal malformations $(n=38)$, stillbirths $(n=64)$, neonates $(n=172)$, infants $(n=88)$, and children less than 18 -year-old $(n=69)$ (Fig. 1). Consanguinity was documented in the majority $(n=449)$. Positive family history was noted in 340, while the rest were simplex. CMA was performed at baseline for all simplex cases. Those with negative CMA results as well as all familial cases underwent the following testing strategies: index-only ES ( $n=$ $341)$, duo ES on the parents $(n=42)$, index-only panel $(n=67)$, duo panel on the parents $(n=14)$, autozygosity mapping and targeted sequencing $(n=12)$, whole-genome sequencing $(n=1)$, and RNA-seq $(n=1)$ (Fig. 1$)$.

\section{Higher yield compared to regular genetic diagnosis}

A likely causal variant was identified in 63.8\% $(n=$ $307 / 481$, solved), and in 50 cases (10.3\%), the candidate was a variant of uncertain significance (ambiguous), while no candidate variant was identified in 124 (25.7\%, negative) (Fig. 1 and Additional file 1: Table S1). The percentage of solved cases is significantly higher than what we published for general diagnostic exomes based on $>2200$ families from the same population (307/481 (63.8\%) vs. 960/2219 (43.2\%), $p<$ $0.0001)$ [14]. This may be in part due to a higher percentage of loss of function (LOF) variants in this cohort compared to the general diagnostic cohort (187/ $307(60.9 \%)$ vs. $470 / 961$ (48.9\%), $p=0.0006)$. Indeed, the percentage of negative cases is comparable between the two cohorts (124/481 (25.7\%) vs. 602/2219 $(27 \%), p=0.28)$, which suggests that the higher solve rate is primarily driven by a larger P/LP to VUS ratio. Of note, the yield was higher among consanguineous families (294/449 (65.4\%) vs. $13 / 32(40 \%))$ and in certain phenotypic categories, e.g., $78 \%$ among terminated pregnancies (Additional file 1: Table S1 and Additional file 3: Table S2).

\section{A}

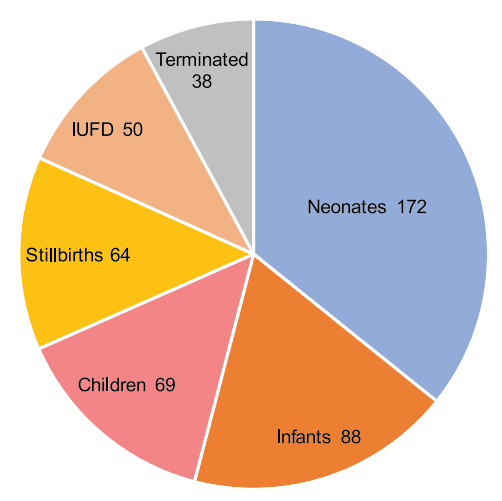

C

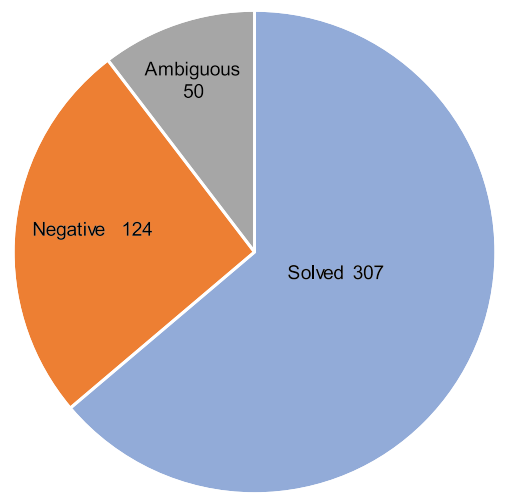

\section{$\mathrm{B}$}

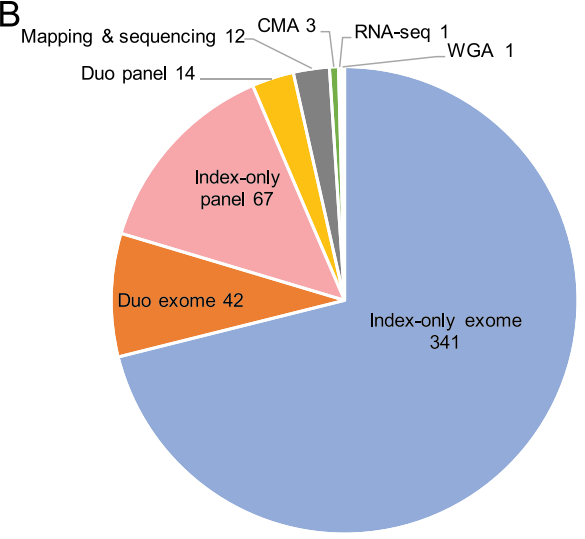

D

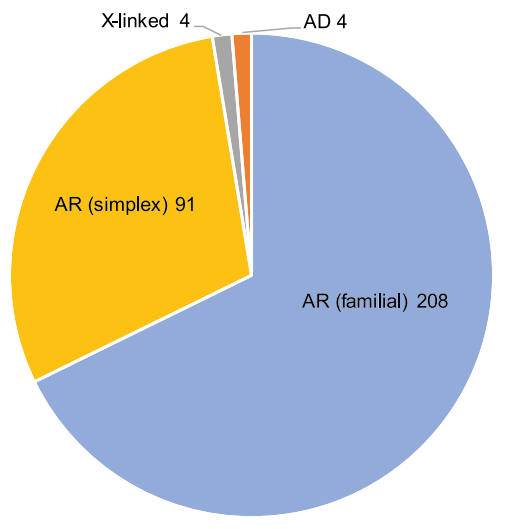

Fig. 1 Pie chart summary of the study. A Age at death of the study cases. B Molecular tools used for the analysis in the study. C Molecular outcome of the study. $\mathbf{D}$ Inheritance pattern of the variants (pathogenic and likely pathogenic) identified in the study 


\section{Predominance of autosomal recessive trait-associated} biallelic variants

Although CMA was performed on all simplex cases $(n=$ 141 ), a causal variant was identified in the form of chromosomal deletion in only three cases (14DG1134, 16DG1465, and 15DG0267); the latter is a case of holoprosencephaly linked to a large homozygous deletion that encompasses ZIC2 and ZIC5 (Additional file 1: Table S1, Additional file 2: Figure S3 A-B). On the other hand, exome and panel sequencing revealed dominant (de novo) variants (ATP1A3 and $N A L C N)$ in three cases and X-linked variants $(A T P 11 C$, $P D H A, M S N$, and $F A A H 2$ ) in four cases. In comparison, autosomal recessive variants accounted for the overwhelming majority of solved cases (96.7\%, 297/307). Importantly, 30.9\% $(92 / 297)$ of the solved cases with recessive variants lacked family history, i.e., simplex (Fig. 1), which highlights the importance of autosomal recessive variants in consanguineous populations even in the setting of negative family history.

\section{Discovery and support of disease gene candidates}

Compelling variants were identified in 46 genes with no established link to Mendelian phenotypes at the time of analysis (Additional file 3: Table S3). Of particular interest is EHBP1L1 because it was independently identified in two families with non-immune hydrops fetalis (NIHF) resulting in recurrent fetal loss. The first case 14DG1037 was found to be homozygous for EHBP1L1: NM 001099409.3:c.3333_3346delinsAGAGTCAGTAGCA:

p.(Arg1112Glufs*20) while the second family was analyzed by the duo exome of the parents [14], who were found to be heterozygous for the splice variant NM_001099409: c.4004-1G $>$ A that resulted in skipping of exon 14 and the creation of an aberrant transcript (Fig. 2). EHBP1L1 was identified as a novel binding protein of Rab8, a highly conserved Rab guanosine triphosphatase (GTPase) that plays an essential role in exocytosis toward the polarized plasma membrane [15]. Indeed, deficiency of EHBP1L1, like that of Rab8, results in the lysosomal accumulation of apical but not basolateral membrane proteins. Furthermore, Ehbp1l1-/- die within 24h of birth although very few details are described about their pathology apart from abnormal intestinal microvilli [15]. Another line described by DMDD (https://dmdd.org.uk/mutants/Ehbp1l1) showed that $E h b p 1 l 1^{-/}$is lethal with subcutaneous edema,

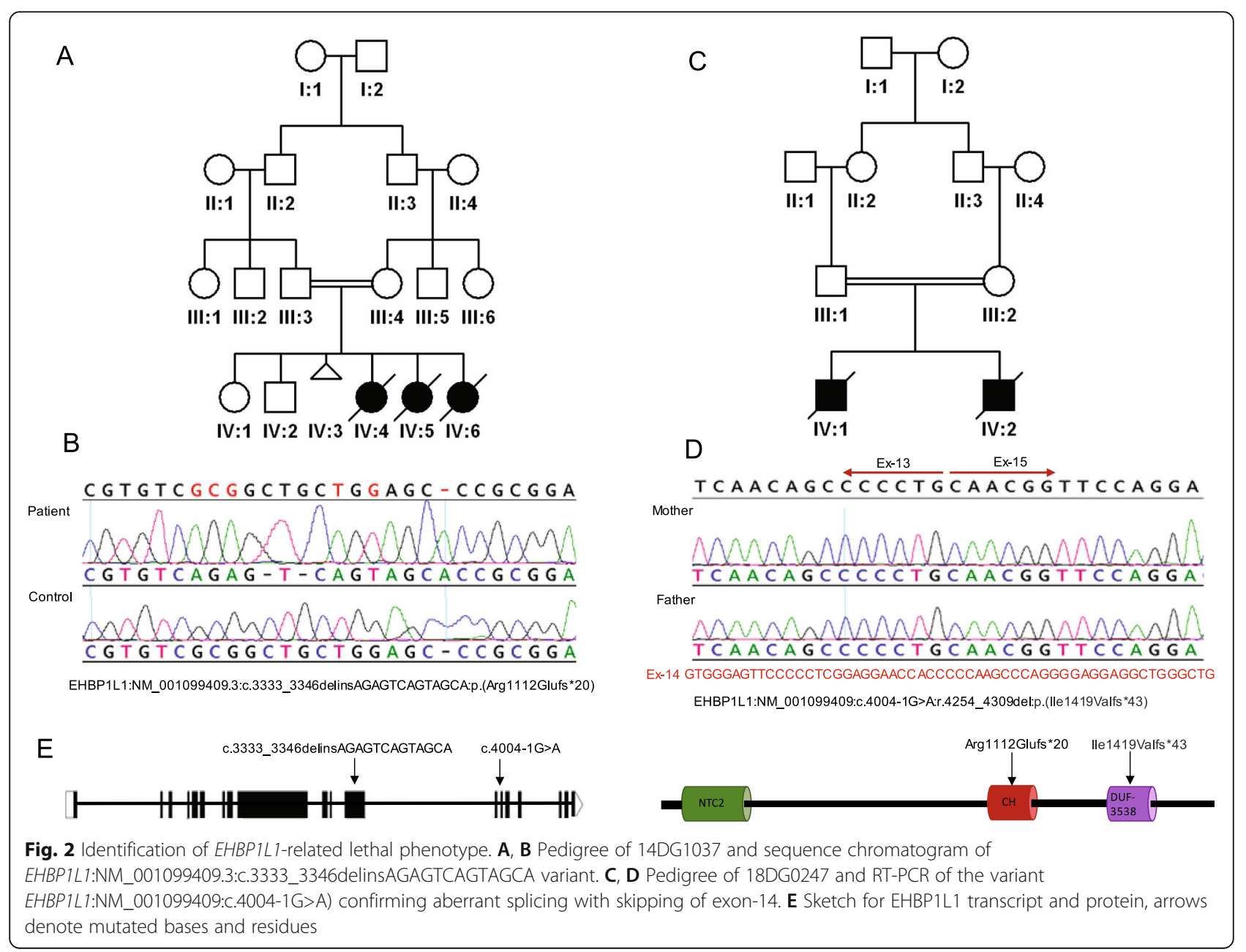




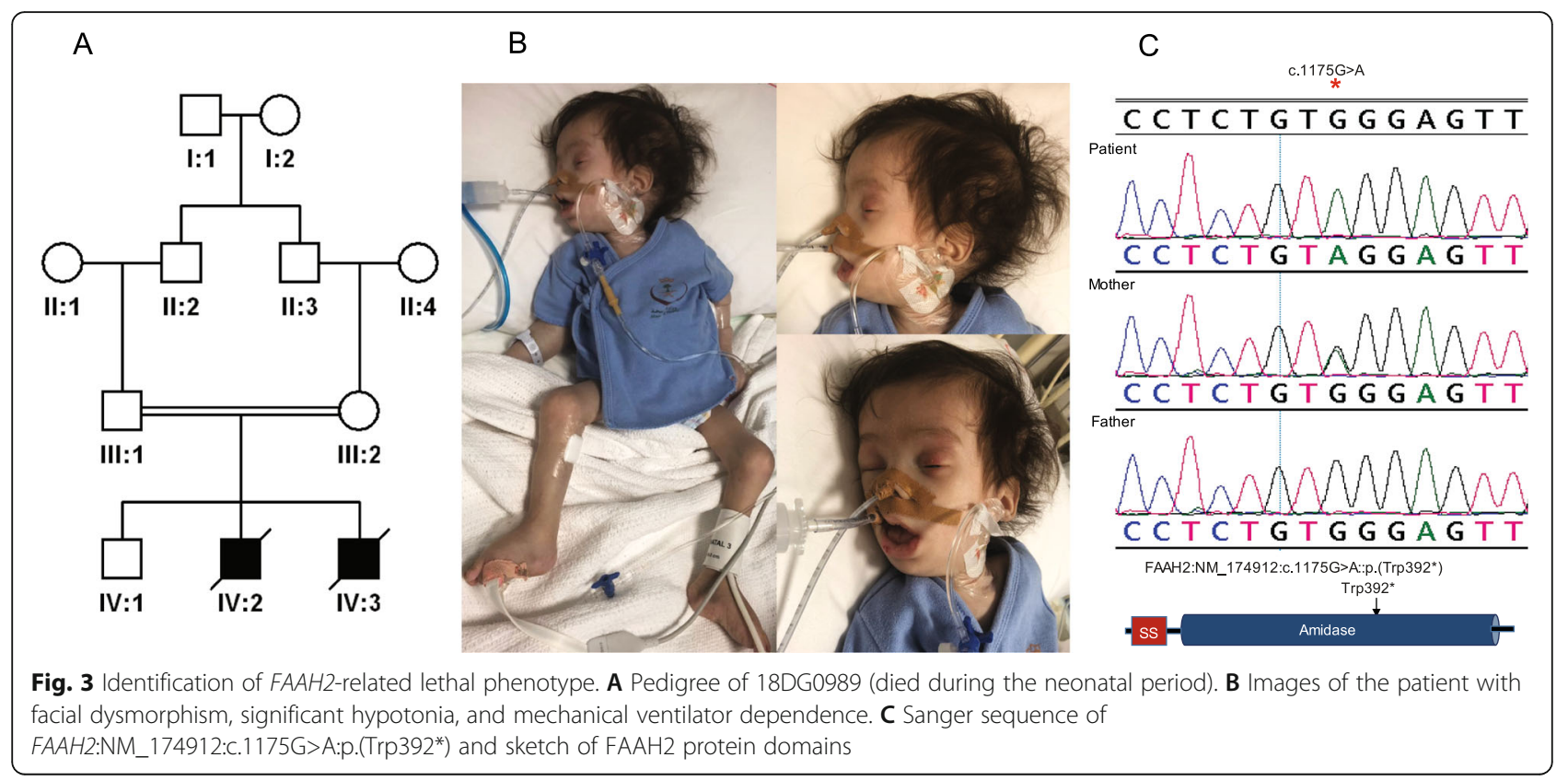

perimembraneous ventricular septic defect, and thin myocardium.

We also provide supporting evidence of the previously published candidacy of two other genes. Patient 18DG0989 presented with a classical Zellweger syndrome phenotype including global developmental delay, seizure disorder, severe hypotonia, failure to thrive, adrenal insufficiency and elevated very long-chain fatty acids and liver enzymes (Fig. 3 and Additional file 3: Table S4). He was found to be hemizygous for FAAH2: NM_174912.3:c.1175G>A:p.(Trp392*) inherited from the normal mother. FAAH2 was first proposed as a novel candidate disease-related gene by Sirrs et al. who described a male with a neurodevelopmental disorder characterized by neonatal hypotonia, epilepsy, autism, and white matter abnormalities [16]. Additional cases were reported by others albeit with few clinical details [17, 18]. Again, there remains no phenotypic listing for FAAH2 in OMIM. A second example is patient 16DG0125 who died as a young child of severe respiratory infections, complicated by pulmonary hypertension and progressive occlusion of pulmonary veins although

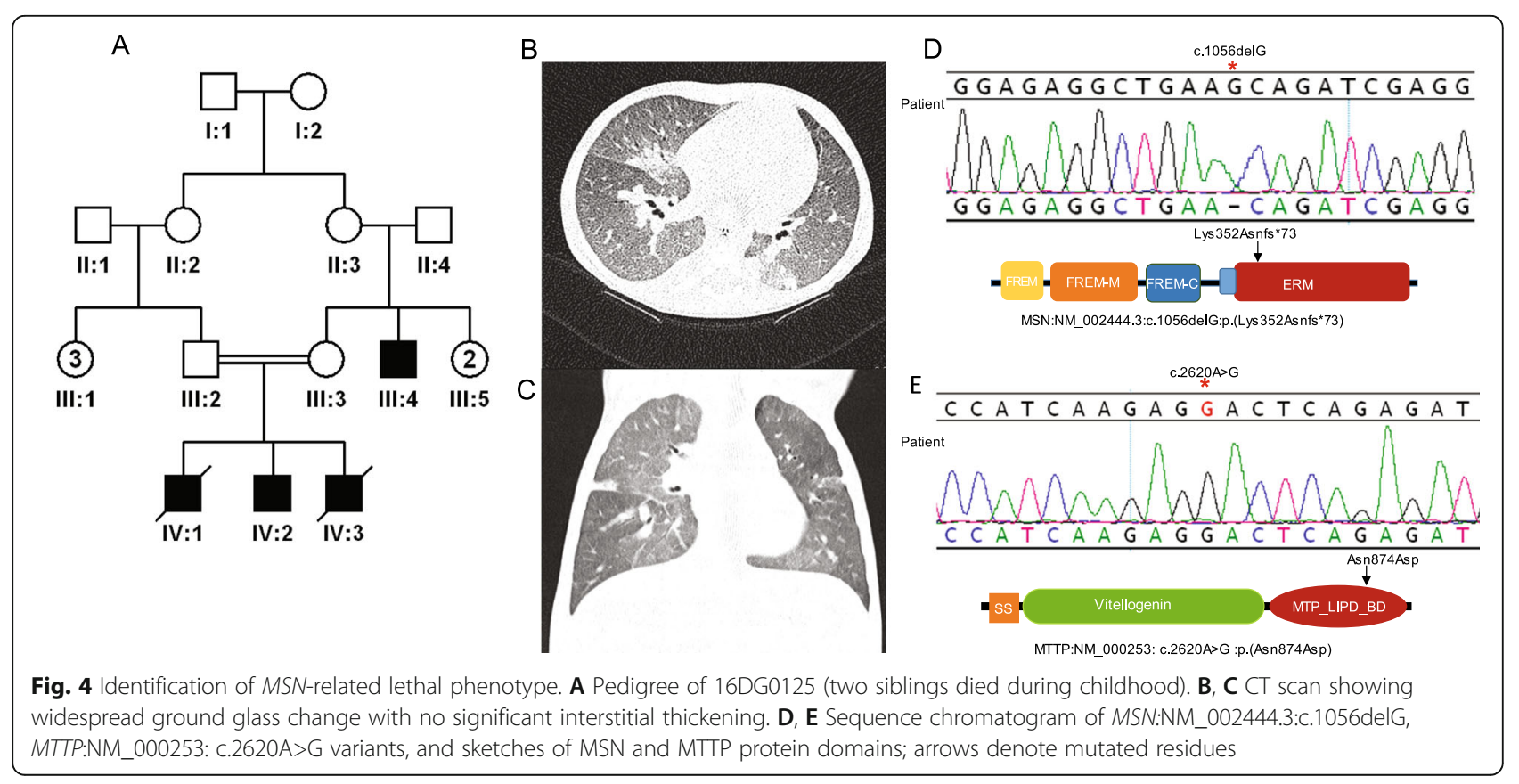


no underlying etiology was identified (Fig. 4). We identified a hemizygous truncating variant in MSN (NM 001931.3:c.975G>A:p.(Lys352Asnfs"73)), which was proposed as a candidate gene for immunodeficiency based on a single publication [19]. Interestingly, the same maternally inherited variant was also found in a brother and a maternal cousin who are both still alive and healthy, which is consistent with the highly variable age of onset of $M S N$-related immunodeficiency [19].

\section{Phenotypic expansion}

As expected for a cohort with lethal phenotypes, we encountered instances of lethal presentations of genes typically linked to much milder phenotypes. Perhaps the most striking is the family with FZD6 variant alleles. Although the only phenotype linked to this gene in OMIM is a naillimited abnormality, we have previously described a family with NIHF and proposed FZD6 as the likely candidate [20]. The second family we describe here is one of three families with FZD6-related NIHF that will be published elsewhere (Sheppard et al., manuscript in preparation). Remarkably, this family has multiple members with the classical FZD6related nail disorder with history of NIHF during pregnancy, which suggests that FZD6-related NIHF, like THSD1-related NIHF $[4,20]$, can be compatible with longterm survival if mortality in the newborn period is avoided. Additional examples include case 09DG01414 with a homozygous truncating variant in TMPRSS15 (NM 002772.2:c.2808_2809insATCA p.(Ser937Ilefs*4)) who died in infancy, an unusual presentation of enterokinase deficiency. Similarly, case 21DG0001 with a homozygous truncating variant in TNNT3 (NM_006757:c.723-2A>G) presented with severe non-immune hydrops fetalis and neonatal death (Additional file 2: Fig. S5), which have not been reported to date in TNNT3-related myopathy. In the same context, duo ES for parents of a deceased neonate (19DG0461) with a history of fetal akinesia and polyhydramnios revealed the shared carrier status of a splice variant in TTN (NM_001267550.2:c.28462+1G>T), which likely explains the phenotype of the index patient in the homozygous state as reported by others [21-23].

We also highlight case 17DG0032 whose lethal NeuLaxova syndrome-like presentation represents a phenotypic expansion compared to the four patients reported to date with the lethal LARS2-related HLSA (hydrops, lactic acidosis, and sideroblastic anemia) syndrome (Altawil et al., manuscript in preparation and $[24,25]$ ).

\section{Variants in "dominant" genes that are lethal in the biallelic state}

We observed two classes of lethal variants in genes that are typically linked to dominant phenotypes only. First, there are recessive variants with no apparent clinical consequences in the carrier parents but result in a lethal phenotype in the offspring. Patient 16DG1276 with a homozygous LZTR1 variant and NIHF (Additional file 2: Fig.S6) was proposed as the first instance of a truly recessive inheritance of this Noonan syndrome-linked gene, which was later confirmed by others [4, 26]. Similarly, although MYH11 has been tentatively linked to adult-onset aortic aneurysm, we have one case (17DG1094) with a homozygous truncating variant (NM_022844:exon9:c.1033+1G>A) and had a distinct fetal presentation (Additional file 2: Fig.S7) consistent with several reported megacystismicrocolon syndrome cases caused by biallelic MYH11 variants $[14,27]$. Currently, aortic aneurysm is the only phenotype listed by OMIM under MYH11. Similarly, homozygous variants in FBN2 and KRIT1, linked to autosomal dominant congenital contractural arachnodactyly and cerebral cavernous malformations 1, respectively, inherited from healthy heterozygous parents were identified in patients with arthrogryposis and NIHF, respectively. Furthermore, a previously reported likely pathogenic BMPR $1 A$ variant was found to be shared by normal consanguineous parents who presented for prenatal counseling regarding recurrent skeletal dysplasia and IUFD (20DG1384). We propose this to be a novel recessive phenotype of BMPR1A, a gene hitherto linked to autosomal dominant intestinal polyposis, which would be in line with our previous finding of a novel recessive phenotype of APC comprising severe bone malformations [28, 29].

The second class is dominant variants that were rendered homozygous by the union of two affected heterozygous parents, i.e., semidominant inheritance. For example, the pregnancy of 16DG0393 was terminated because of the identification of a homozygous PKD1 pathogenic variant resulting in severe polycystic kidneys and posterior fossa abnormality consistent with the published literature, while the consanguineous asymptomatic parents were found in retrospect to have autosomal dominant polycystic kidney disease [30, 31]. Similarly, patient F8317 died of severe skeletal dysplasia caused by a known achondroplasia-related FGFR3 variant inherited from both affected parents (Additional file 2: Fig. S8).

\section{Extreme phenotypic variability of some variants depending on the genotype}

Family 15DG2154 presented with the lethal microcephalymicromelia syndrome in multiple pregnancies, which was mapped to a severe splicing variant in DONSON, the same variant that when inherited in trans with milder variants results in a much milder Seckel syndrome as described previously [32] (a range of DONSON-related phenotypes has also been described [33]). A similar scenario is found in 20DG0807 with a homozygous variant in CHAT resulting in IUFD, while compound heterozygosity for the same variant with a milder variant was reported to result in a viable congenital myasthenic phenotype [34]. 


\section{Implications for MAF interpretation}

In patient 15DG0936 with a lethal form of hepatic ciliopathy, we initially dismissed the candidate variant TTC26: NM_001144923.2:c.4-1G>C because of its relatively high local population frequency (MAF:0.002); however, it was proven causal on RT-PCR as previously described [35]. This frequency should predict a more common contribution of this founder variant to our molecular autopsy cohort than observed. This discrepancy may be explained by the success of liver transplant in preventing death among affected children. This founder variant is one of 100 founder variants that explained nearly half of the solved cases $161 / 307$ (52.4\%). Interestingly, most of the founder variants $(67 \%(67 / 100))$ are unique to the local population, while the rest were encountered in other world populations (Additional file 3: Table S5).

\section{The potential of other "omics" in molecular autopsy}

In addition to the overrepresentation of conspicuous LOF variants in our cohort, cryptic LOF variants were also observed. One example is patient 13DG1520 who died as a neonate and initially had negative exome results. However, reanalysis revealed a homozygous synonymous variant in DLAT (NM_001931.3:c.975G>A:p.(= )) [36]. RT-PCR confirmed the splicing nature of this variant (r.788_975del: p.(Phe264Glyfs*32), which was the last nucleotide of exon 6 and was, therefore, reclassified as LOF. Interestingly, this case also represents a phenotypic expansion of DLAT-related pyruvate dehydrogenase deficiency. A more challenging case was family 11DG1647 that lost several children with severe neonatal lactic acidosis, which could not be solved by exome sequencing. Positional mapping allowed us to prioritize a variant that had been dismissed because it was synonymous in an apparently non-splicing position (BCS1L:NM_ 001079866:exon3:c.441C>T:p.F147F). RT-PCR using parental samples, however, revealed that this is a strong exonic splicing variant and confirmed its LOF nature (Additional file 2: Fig. S9). Although these cases were solved by targeted RT-PCR, they highlight the potential role of transcriptomics to supplement exome sequencing at least in select cases. Indeed, in family 16DG1465 that lost four children with Hirschsprung disease, gastroesophageal reflux disease, coarse facial features, severe global developmental delay, agenesis of the corpus callosum, failure to thrive, and cataract, exome sequencing was negative but RNA-seq as described before [36] highlighted an abnormal profile of EPG5 and subsequent analysis revealed a homozygous deletion of exon 1 (Fig. 5).

\section{Multi-locus pathogenic variation: families with multiple lethal phenotypes}

In the above described patient 16DG0125 with a hemizygous truncating variant in $M S N$, we also identified a homozygous variant in MTTP (NM_000253:c.A2620G: p.Asn874Asp) that may have contributed to his demise due to severe diarrhea (Fig. 4). On the other hand, we report one family that lost two pregnancies due to NIHF, and a different homozygous variant was identified in each: 13DG0259 was homozygous for FCRL4: NM_ 031282:c.847+1G>A while 15DG0933 was homozygous for SLC17A5:NM_012434:c.1111+1G>A. This has important implications on the utility of segregation analysis of lethal variants in inbred families and a reminder of the residual risk for additional autosomal recessive traits in consanguineous families that present for counseling regarding one autosomal recessive trait [37].

\section{Discussion}

In this cohort, we describe our experience with lethal variants identified by molecular autopsy in young patients with suspected genetic etiology. As expected for a population enriched for consanguinity [38, 39], the overwhelming majority of variants were autosomal recessive, even when positive family history was lacking. In comparison, only 5 out of 9 variants identified by QuinlanJones et al. in their smaller study with a narrower definition of molecular autopsy (lethal malformations during pregnancy) were recessive [5]. Similarly, the study by Stanley et al. on genetic causes of stillbirths observed that only $20 \%$ of the causal variants were recessive [40]. Thus, the two ways in which lethal variants can circumvent the reproduction barrier because of their low reproductive fitness, i.e., to arise de novo (dominant) or to pass to the next generation in the monoallelic state (recessive), can be observed in action albeit to a variable degree depending on the population structure, e.g., inbred vs. outbred [41].

Despite the heterogeneous nature of this cohort, the lethal nature of the causal variants represents a common denominator that we think contributes to the high diagnostic rate. In other words, it is likely that lethal variants are more likely to belong to classes that are more readily identified and classified as pathogenic or likely pathogenic, and this is supported by our finding that this cohort is enriched for LOF variants (see below). Consistent with this notion, a higher than average diagnostic rate has also been reported in ES sequencing of children with severe phenotypes in intensive care units [42]. The detailed listing of phenotypes in our cohort should allow comparison to other studies that focused on specific phenotypes. For example, the yield among pregnancy terminations for fetal malformations was $78 \%$ in this cohort (Additional file 3: Table S2 ) compared to 20\% (74\% if ambiguous cases were also included) by others [43].

We have taken advantage of this predominance of autosomal recessive variants to identify the likely cause 


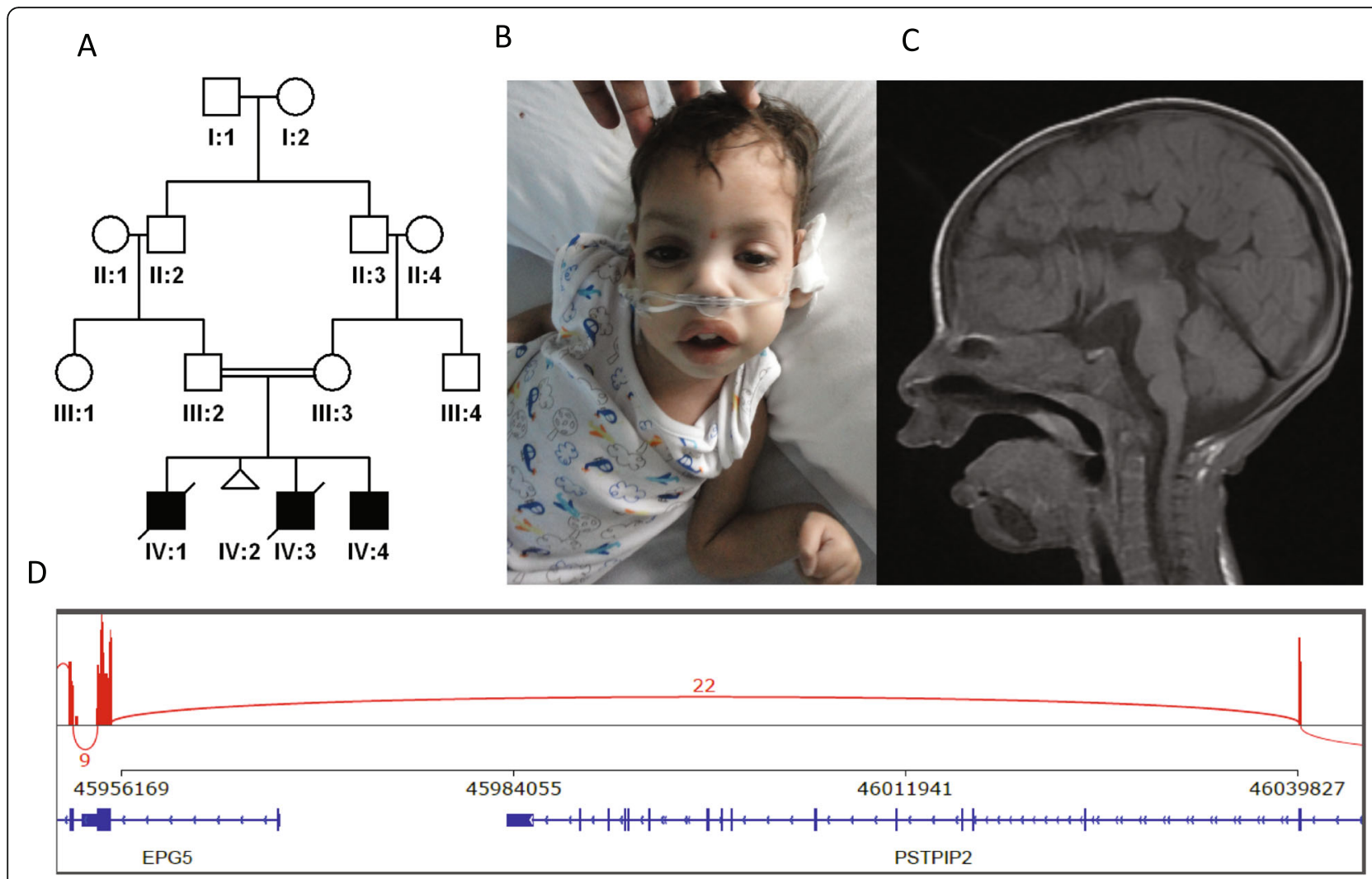

Fig. 5 RNA-seq identifies EPG5-related lethal phenotype. A, Pedigree of 16DG1465 with deletion of EPG5 exon-1 (two siblings died during infancy). B Image of the patient with facial dysmorphism. C MRI showing absent corpus callosum. D A sashimi plot showing the base-level density of reads mapped to a genomic region surrounding the $\sim 70 \mathrm{~K}$ deletion site. The $x$-axis represents the genomic coordinate in hg38. The $y$-axis represents per-base read counts. On the bottom, the boxes are annotated exons, the horizontal lines are introns, and the left-facing arrowheads indicate the negative strand. Arcs connecting exons represent splice junction reads. The plot shows that no reads are mapped to exon 1 of EPG5 and that 22 splitreads are mapped to an aberrant splicing junction between exon 2 of PSTPIP2 and exon 2 of EPG5 that are 84Kbp apart

of death when no DNA sample from the deceased individual is available by studying the presumptive carrier parents. This "molecular autopsy by proxy" approach has proven helpful in the majority of families who could now receive accurate genetic counseling and make informed reproductive choices. Unlike the de novo paradigm, the recurrence risk in the autosomal recessive paradigm is high, which underscores the clinical utility in making a precise molecular diagnosis that can be the basis for such preventative measures as cascade carrier testing, prenatal diagnosis, and preimplantation genetic testing [44]. Although our focus in molecular autopsy by proxy was on recessive variants, we should note the important contribution of balanced chromosomal rearrangements in asymptomatic parents to lethal phenotypes among the unbalanced offspring. Dong et al. found that $11.7 \%$ of couples with recurrent miscarriage harbor balanced rearrangements many of which could only be identified using genome sequencing [45]. This suggests that future studies into molecular autopsy by proxy should consider screening parents for both SNVs and CNVs (including balanced rearrangements) to maximize the yield.

The enrichment for LOF in lethal variants has been noted by others [40]. This may have contributed to the high yield of the molecular autopsy by enabling more variants to be classified as pathogenic or likely pathogenic. Several points are worth highlighting in this regard. First, not all LOF are readily identifiable, especially transcript-deleterious variants that masquerade as benign synonymous variants in non-canonical exonic splice sites. Although positional mapping has proven very helpful in highlighting these variants [46], we acknowledge that this is not always feasible. On the other hand, RNAseq has been shown to effectively identify and properly classify such variants [36], so it is likely to have a prominent role in future molecular autopsy studies as shown in the example described here. Second, there is a growing appreciation of genetic disorders that are only caused by a combination of mild and severe variants in trans because homozygosity for the mild variants may be 
asymptomatic while homozygosity for the severe variant is embryonically lethal. Molecular autopsy in consanguineous populations is an ideal setup for the latter class of LOF as shown in the example involving the DONSON variant. This is a reminder that the clinical interpretation of a variant must take its genotype into consideration because a variant may be lethal in one context but not others. Third, caution must be exercised when interpreting LOF variants in genes linked to dominant conditions when these are encountered in asymptomatic individuals. The tendency to rule these as examples of nonpenetrance should be balanced by the possibility that these may represent bona fide autosomal recessive inheritance, which has a very different recurrence risk estimate [47]. Lastly, the use of population frequency to interpret the pathogenicity of an apparently LOF variant can be deceiving unless a database of lethal variants is established from the same population.

"Embryonic lethal genes" is a term used commonly in model organisms and has been useful in highlighting genes that are likely to be disease-related in humans as shown in the mouse and fruitfly [48, 49]. However, this term overlooks the unlimited "allelic series" of the corresponding genes in humans and the remarkable phenotypic diversity thereof. This is why it is difficult to have an accurate estimate of the percentage of "embryonic lethal genes" in humans in comparison to the available estimates from model organisms. Even in this molecular autopsy cohort where the variants are by definition lethal, death during fetal development was only observed in a subset of cases. PHGDH is a case in point. Phgdh -/- mice die during embryonic development while in humans variants in this gene can result in phenotypes ranging from stillbirths due to Neu-Laxova syndrome to the much milder serine deficiency-related neurodevelopmental disorder [50,51]. It should also be noted that embryonic lethality may even precede biochemical pregnancy as demonstrated in TLE6 that has been linked to the earliest possible form of embryonic lethality, involving failure of zygote cleavage, and other variants that have been shown to cause embryonic lethality that can only be identified through IVF clinics [52-54].

Delineating the severe end of the phenotypic expression of Mendelian genes through a broadly defined molecular autopsy approach such as the one implemented in this work can contribute significantly to the clinical annotation of the human genome both at the gene and variant levels. One particularly interesting area of research in population genomics is inferring recessive lethal variants by calculating their depletion in the control population in the biallelic state. The short-read sequencing technology that is typically used in generating available variant databases precludes phasing of most of the rare variants such that depletion of homozygotes is the only practical alternative. However, as the frequency of a variant decreases, the population size required to confidently conclude its depletion in the homozygous state becomes exponentially and impractically larger except in consanguineous populations. Indeed, we note that $36.7 \%$ $(132 / 359)$ of the lethal variants described here exist in gnomAD albeit at such low frequencies that it could not be concluded if they are lethal in the recessive state and yet they were identified in our population despite a comparably low MAF. The one exception (TTC26:NM 001144923.2:c.4-1G>C) where the local MAF was high and did not reflect a correspondingly strong representation in the molecular autopsy cohort is a reminder that control databases from a given population afford a limited interpretation power unless they are supplemented by efforts to catalog lethal variants from the same population. It is hoped that this and future molecular autopsy from other populations will help toward that goal.

\section{Conclusions}

We conclude that molecular autopsy is a powerful tool to reveal the cause of death when applied correctly. Novel clinical and biological insights can be gained from this approach, the clinical utility of which, especially when broadly defined, is becoming increasingly clear. In the future, it is likely that molecular autopsy will involve other omics in addition to genome sequencing. The value of molecular autopsy in defining lethal variants in humans on the clinical annotation of the human genome cannot be overstated.

\section{Supplementary Information}

The online version contains supplementary material available at https://doi. org/10.1186/s13073-021-00973-0.

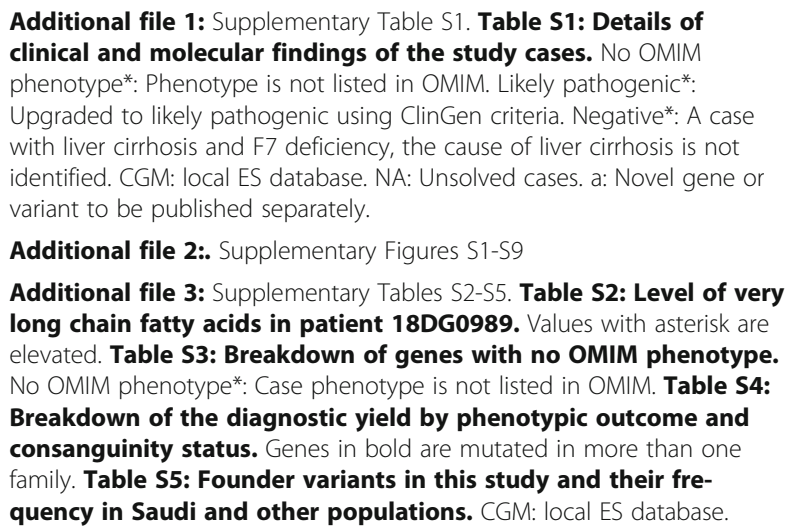

Additional file 2:. Supplementary Figures S1-S9

Additional file 3: Supplementary Tables S2-S5. Table S2: Level of very long chain fatty acids in patient 18DG0989. Values with asterisk are elevated. Table S3: Breakdown of genes with no OMIM phenotype. No OMIM phenotype*: Case phenotype is not listed in OMIM. Table S4: Breakdown of the diagnostic yield by phenotypic outcome and consanguinity status. Genes in bold are mutated in more than one family. Table S5: Founder variants in this study and their frequency in Saudi and other populations. CGM: local ES database.

\section{Acknowledgements}

We thank the study families for their enthusiastic participation. LA was supported by "Researchers Supporting Project number (RSP-2021/181), King Saud University, Riyadh, Saudi Arabia." XG was supported by "King Abdullah University of Science and Technology (KAUST) Office of Sponsored Research (OSR) under award numbers FCS/1/4102-02-01, URF/1/4098-01-01, REI/1/ 4216-01-01, REl/1/4437-01-01, REI/1/4473-01-01, and REI/1/4742-01-01." 
Molecular Autopsy Consortium

Hesham Aldhalaan ${ }^{1}$, Abdullah Alfaifi ${ }^{2}$, Amal Alhashem ${ }^{4}$, Khalid Alhasan ${ }^{3}$, Maha Alnemer ${ }^{5}$, Nada Alsahan ${ }^{5}$, Suad Alyamani ${ }^{1}$, Hamad Alzaidan ${ }^{6}$, Kerr Brownyn ${ }^{7}{ }^{12}$, Angela Condie ${ }^{8}$, Eissa Faqeih ${ }^{9}$, Rubina Khan ${ }^{5}$, Wesam Kurdi ${ }^{5}$ Osama Muthaffar ${ }^{10}{ }^{15}$, Amira Nabil ${ }^{11}$, William Newman ${ }^{712}$, Mohammad M. Al-Qattan ${ }^{13}$, Zuhair Ráhbeeni ${ }^{6}$, Rawda Sunbul ${ }^{14}$, Mohammad H. Temsah ${ }^{3}$, Maha Tulbah ${ }^{5}$, Mohammed Zain ${ }^{2}$.

${ }^{1}$ Department of Neurosciences, King Faisal Specialist Hospital and Research Center, Riyadh, Saudi Arabia

${ }^{2}$ Department of Pediatrics, Security Forces Hospital, Riyadh, Saudi Arabia

${ }^{3}$ Department of Pediatrics College of Medicine, King Saud University

${ }^{4}$ Department of Pediatrics, Prince Sultan Military Medical City, Riyadh, Saudi Arabia.

${ }^{5}$ Department of Obstetrics and Gynecology, King Faisal Specialist Hospital

and Research Centre, Riyadh, Saudi Arabia.

${ }^{6}$ Department of Medical Genetics, King Faisal Specialist Hospital and

Research Center, Riyadh, Saudi Arabia.

${ }^{7}$ Evolution and Genomic Sciences, School of Biological Sciences, Faculty of

Biology, Medicine and Health, University of Manchester, Manchester, UK

${ }^{8}$ Bach Christian Hospital, Qalandarabad, Abbottabad, Hazara, KPK, Pakistan.

${ }^{9}$ Department of Pediatric Subspecialties, Children's Hospital, King Fahad

Medical City, Riyadh, Saudi Arabia.

${ }^{10}$ Department of Pediatrics, King Abdulaziz University, Jeddah, Saudi Arabia

${ }^{11}$ Human Genetics department, Medical Research Institute, Alexandria University, Egypt.

${ }^{12}$ Manchester Centre for Genomic Medicine, Manchester University NHS Foundation Trust, Manchester, UK

${ }^{13}$ Department of Surgery, King Saud University, Riyadh, Saudi Arabia

${ }^{14}$ Department of Pediatrics, Qatif Central Hospital, Qatif, Saudi Arabia.

${ }^{15}$ Department of Pediatrics, King Faisal Specialist Hospital and Research

Center, Jeddah, Saudi Arabia

\section{Authors' contributions}

Data acquisition, analyses, and interpretation, HS, LA, SM, HA, and FA: recruitment, sample preparation, $P C R$, and Sanger sequencing, $N E, M H, F A$, and OA; RNA-seq analysis and interpretation, HK and XG. FSA and all authors: manuscript draft and writing. Molecular Autopsy Consortium: referring physicians provided clinical information and relevant images. All read and approved the final manuscript. Study supervision: FSA.

\section{Funding}

LA was supported by "Researchers Supporting Project number (RSP-2021/ 181), King Saud University, Riyadh, Saudi Arabia."

XG was supported by "King Abdullah University of Science and Technology (KAUST) Office of Sponsored Research (OSR) under award numbers FCS/1/ 4102-02-01, URF/1/4098-01-01, REI/1/4216-01-01, REI/1/4437-01-01, REI/1/ 4473-01-01, and REI/1/4742-01-01."

\section{Availability of data and materials}

All variants' data has been deposited to ClinVar and that ClinVar accession IDs are provided in Additional file 1: Supplementary Table S1. RNA-seq data relevant to the 16DG1465 case have been deposited in NCBI Sequence Read Archive under bioproject ID PRJNA761889 (http://www.ncbi.nlm.nih.gov/ bioproject/761889) [55]. Due to local IRB (King Faisal Specialist Hospital and Research Center Research Advisory Council) regulations to protect the privacy of human subjects, individual-level raw data cannot be deposited in public databases.

\section{Declarations}

\section{Ethics approval and consent to participate}

This study was approved by King Faisal Specialist Hospital and Research Center - Research Ethics committee. The research conformed to the principles of the Helsinki Declaration. All patients/participants or their legal guardians gave their written informed consent to participate in the study.

\section{Consent for publication}

Written informed consent for publication of clinical details was obtained from the participants or legal guardians. This study was cleared for publication by the Office of Research Affairs (Publication Number 2215236).

\section{Competing interests}

The authors declare that they have no competing interests.

\section{Author details}

${ }^{1}$ Department of Translational Genomics, Center for Genomic Medicine, King Faisal Specialist Hospital and Research Center, Riyadh, Saudi Arabia. 2Department of Zoology, College of Science, King Saud University, Riyadh, Saudi Arabia. ${ }^{3}$ Center of Excellence for Biomedicine, King Abdulaziz City for Science and Technology, Riyadh 12354, Saudi Arabia. ${ }^{4}$ Computational Bioscience Research Center (CBRC), King Abdullah University of Science and Technology (KAUST), Thuwal, Saudi Arabia.

Received: 21 May 2021 Accepted: 17 September 2021 Published online: 13 October 2021

References

1. Burton JL, Underwood J. Clinical, educational, and epidemiological value of autopsy. Lancet. 2007;369:1471-80.

2. Ackerman, M.J., Tester, D.J., and Porter, C.B.J. (1999). Swimming, a genespecific arrhythmogenic trigger for inherited long QT syndrome. In Mayo Clinic Proceedings. (Elsevier), pp 1088-1094.

3. Webster G, Puckelwartz MJ, Pesce LL, Dellefave-Castillo LM, Vanoye CG, Potet $F$, et al. Genomic autopsy of sudden deaths in young individuals. JAMA Cardiol. 2021. https://doi.org/10.1001/jamacardio.2021.2789.

4. Shamseldin HE, Kurdi W, Almusafri F, Alnemer M, Alkaff A, Babay Z, et al. Molecular autopsy in maternal-fetal medicine. Genet Med. 2018:20:420-7.

5. Quinlan-Jones E, Lord J, Williams D, Hamilton S, Marton T, Eberhardt RY, et al. Molecular autopsy by trio exome sequencing (ES) and postmortem examination in fetuses and neonates with prenatally identified structural anomalies. Genet Med. 2019;21:1065-73.

6. Rancati G, Moffat J, Typas A, Pavelka N. Emerging and evolving concepts in gene essentiality. Nat Rev Genet. 2018;19:34

7. Dickinson ME, Flenniken AM, Ji X, Teboul L, Wong MD, White JK, et al. Highthroughput discovery of novel developmental phenotypes. Nature. 2016.

8. Amorim CEG, Gao Z, Baker Z, Diesel JF, Simons YB, Haque IS, et al. The population genetics of human disease: the case of recessive, lethal mutations. PLoS Genet. 2017:13:e1006915.

9. Monies D, Abouelhoda M, AlSayed M, Alhassnan Z, Alotaibi M, Kayyali H, et al. The landscape of genetic diseases in Saudi Arabia based on the first 1000 diagnostic panels and exomes. Hum Genet. 2017.

10. Richards S, Aziz N, Bale S, Bick D, Das S, Gastier-Foster J, et al. Standards and guidelines for the interpretation of sequence variants: a joint consensus recommendation of the American College of Medical Genetics and Genomics and the Association for Molecular Pathology. Genet Med. 2015;17:405-23.

11. Karczewski KJ, Francioli LC, Tiao G, Cummings BB, Alfoldi J, Wang Q, et al. Author correction: the mutational constraint spectrum quantified from variation in 141,456 humans. Nature. 2021;590:E53.

12. Mistry J, Chuguransky S, Williams L, Qureshi M, Salazar GA, Sonnhammer ELL, et al. Pfam: the protein families database in 2021. Nucleic Acids Res. 2021:49:D412-9

13. Rehm HL, Berg JS, Brooks LD, Bustamante CD, Evans JP, Landrum MJ, et al. ClinGen--the clinical genome resource. N Engl J Med. 2015:372:2235-42.

14. Monies D, Abouelhoda M, Assoum M, Moghrabi N, Rafiullah R, Almontashiri $\mathrm{N}$, et al. Lessons learned from large-scale, first-tier clinical exome sequencing in a highly consanguineous population. Am J Hum Genet. 2019; 104:1182-201.

15. Nakajo A, Yoshimura, S.-i., Togawa, H., Kunii, M., Iwano, T., Izumi, A., Noguchi, Y., Watanabe, A., Goto, A., and Sato, T. EHBP1L1 coordinates Rab8 and Bin 1 to regulate apical-directed transport in polarized epithelial cells. J Cell Biol. 2016;212:297-306

16. Sirrs S, van Karnebeek CD, Peng X, Shyr C, Tarailo-Graovac M, Mandal R, et al. Defects in fatty acid amide hydrolase 2 in a male with neurologic and psychiatric symptoms. Orphanet J Rare Dis. 2015;10:1-10.

17. Whibley AC, Plagnol V, Tarpey PS, Abidi F, Fullston T, Choma MK, et al. Finescale survey of $X$ chromosome copy number variants and indels underlying intellectual disability. Am J Hum Genet. 2010;87:173-88.

18. Lim ET, Raychaudhuri S, Sanders SJ, Stevens C, Sabo A, MacArthur DG, et al. Rare complete knockouts in humans: population distribution and significant role in autism spectrum disorders. Neuron. 2013;77:235-42.

19. Lagresle-Peyrou C, Luce S, Ouchani F, Soheili TS, Sadek H, Chouteau M, et al $\mathrm{X}$-linked primary immunodeficiency associated with hemizygous mutations 
in the moesin (MSN) gene. J Allergy Clin Immunol. 2016;138:1681-1689. e1688.

20. Shamseldin HE, Tulbah M, Kurdi W, Nemer M, Alsahan N, Al Mardawi E, et al. Identification of embryonic lethal genes in humans by autozygosity mapping and exome sequencing in consanguineous families. Genome Biol. 2015;16:116.

21. Oates EC, Jones K, Donkervoort S, Charlton A, Brammah S, Smith JE 3rd, et al. Congenital titinopathy: comprehensive characterization and pathogenic insights. Ann Neurol. 2018;83:1105-24.

22. Ravenscroft, G., Clayton, J.S., Faiz, F., Sivadorai, P., Milnes, D., Cincotta, R., Moon, P., Kamien, B., Edwards, M., Delatycki, M., et al. (2020). Neurogenetic fetal akinesia and arthrogryposis: genetics, expanding genotype-phenotypes and functional genomics. J Med Genet.

23. Calame DG, Fatih J, Herman I, Akdemir ZC, Du H, Jhangiani SN, et al. Biallelic pathogenic variants in TNNT3 associated with congenital myopathy. Neurol Genet. 2021;7:e589

24. Riley LG, Rudinger-Thirion J, Schmitz-Abe K, Thorburn DR, Davis RL, Teo J, et al. LARS2 variants associated with hydrops, lactic acidosis, sideroblastic anemia, and multisystem failure. JIMD Rep. 2016;28:49-57.

25. Riley LG, Rudinger-Thirion J, Frugier M, Wilson M, Luig M, Alahakoon TI, et al. The expanding LARS2 phenotypic spectrum: HLASA, Perrault syndrome with leukodystrophy, and mitochondrial myopathy. Hum Mutat. 2020;41:1425-34

26. Johnston JJ, van der Smagt JJ, Rosenfeld JA, Pagnamenta AT, Alswaid A, Baker EH, et al. Autosomal recessive Noonan syndrome associated with biallelic LZTR1 variants. Genet Med. 2018;20:1175-85.

27. Maddirevula S, Alzahrani F, Al-Owain M, Al Muhaizea MA, Kayyali HR, AlHashem A, et al. Autozygome and high throughput confirmation of disease genes candidacy. Genet Med. 2019;21:736-42.

28. Al-Qattan MM, Alkuraya FS. Cenani-Lenz syndrome and other related syndactyly disorders due to variants in LRP4, GREM1/FMN1, and APC: insight into the pathogenesis and the relationship to polyposis through the WNT and BMP antagonistic pathways. Am J Med Genet A. 2019;179:266-79.

29. Patel N, Fageih E, Anazi S, Alfawareh M, Wakil SM, Colak D, et al. A novel APC mutation defines a second locus for Cenani-Lenz syndrome. J Med Genet. 2015:52:317-21.

30. Shamseldin HE, Shaheen R, Ewida N, Bubshait DK, Alkuraya H, Almardawi E, et al. The morbid genome of ciliopathies: an update. Genet Med. 2020;22: 1051-60.

31. Al-Hamed MH, Alsahan N, Rice SJ, Edwards N, Nooreddeen E, Alotaibi M, et al. Bialleleic PKD1 mutations underlie early-onset autosomal dominant polycystic kidney disease in Saudi Arabian families. Pediatr Nephrol. 2019;34: 1615-23.

32. Reynolds JJ, Bicknell LS, Carroll P, Higgs MR, Shaheen R, Murray JE, et al. Mutations in DONSON disrupt replication fork stability and cause microcephalic dwarfism. Nat Genet. 2017:49:537-49.

33. Graham A, Bennett AJ, McLean AA, Zammit VA, Brindley DN. Factors regulating the secretion of lysophosphatidylcholine by rat hepatocytes compared with the synthesis and secretion of phosphatidylcholine and triacylglycerol. Effects of albumin, cycloheximide, verapamil, EGTA and chlorpromazine. Biochem J. 1988;253:687-92.

34. Natera-de Benito D, Topf A, Vilchez JJ, Gonzalez-Quereda L, DominguezCarral J, Diaz-Manera J, et al. Molecular characterization of congenital myasthenic syndromes in Spain. Neuromuscul Disord. 2017;27:1087-98.

35. Shaheen $\mathrm{R}$, Alsahli S, Ewida N, Alzahrani F, Shamseldin HE, Patel N, et al. Biallelic mutations in tetratricopeptide repeat domain 26 (intraflagellar transport 56) cause severe biliary ciliopathy in humans. Hepatology. 2020;71: 2067-79.

36. Maddirevula S, Kuwahara H, Ewida N, Shamseldin HE, Patel N, Alzahrani F, et al. Analysis of transcript-deleterious variants in Mendelian disorders: implications for RNA-based diagnostics. Genome Biol. 2020;21:1-21.

37. AlAbdi L, Alrashseed S, Alsulaiman A, Helaby R, Imtiaz F, Alhamed M, et al. Residual risk for additional recessive diseases in consanguineous couples. Genet Med. 2021.

38. Alkuraya FS. Genetics and genomic medicine in Saudi Arabia. Mol Genet Genom Med. 2014;2:369.

39. Alkuraya FS. How the human genome transformed study of rare diseases. In. (nature publishing group. 2021.

40. Stanley KE, Giordano J, Thorsten V, Buchovecky C, Thomas A, Ganapathi M, et al. Causal genetic variants in stillbirth. N Engl J Med. 2020;383:1107-16.
41. Alkuraya FS. 2020 Curt stern award address: a more perfect clinical genome-how consanguineous populations contribute to the medical annotation of the human genome. Am J Hum Genet. 2021;108:395-9.

42. Zhu T, Gong X, Bei F, Ma L, Chen Y, Zhang Y, et al. Application of nextgeneration sequencing for genetic diagnosis in neonatal intensive care units: results of a multicenter study in China. Front Genet. 2020;11:565078,

43. Yates CL, Monaghan KG, Copenheaver D, Retterer K, Scuffins J, Kucera CR, et al. Whole-exome sequencing on deceased fetuses with ultrasound anomalies: expanding our knowledge of genetic disease during fetal development. Genet Med. 2017;19:1171-8.

44. Alkuraya FS. Impact of new genomic tools on the practice of clinical genetics in consanguineous populations: the Saudi experience. Clin Genet. 2013;84:203-8.

45. Wang H, Dong Z, Zhang R, Chau MHK, Yang Z, Tsang KYC, et al. Low-pass genome sequencing versus chromosomal microarray analysis: implementation in prenatal diagnosis. Genet Med. 2020;22:500-10.

46. Shamseldin HE, Maddirevula S, Faqeih E, Ibrahim N, Hashem M, Shaheen R, et al. Increasing the sensitivity of clinical exome sequencing through improved filtration strategy. Genet Med. 2017;19:593-8.

47. Monies D, Maddirevula S, Kurdi W, Alanazy MH, Alkhalidi H, Al-Owain M, et al. Autozygosity reveals recessive mutations and novel mechanisms in dominant genes: implications in variant interpretation. Genet Med. 2017.

48. Meehan TF, Conte N, West DB, Jacobsen JO, Mason J, Warren J, et al. Disease model discovery from 3,328 gene knockouts by the international mouse phenotyping consortium. Nat Genet. 2017;49:1231-8.

49. Yamamoto S, Jaiswal M, Charng W-L, Gambin T, Karaca E, Mirzaa G, et al. A drosophila genetic resource of mutants to study mechanisms underlying human genetic diseases. Cell. 2014;159:200-14.

50. Shaheen R, Rahbeeni Z, Alhashem A, Faqeih E, Zhao Q, Xiong Y, et al. NeuLaxova syndrome, an inborn error of serine metabolism, is caused by mutations in PHGDH. Am J Hum Genet. 2014;94:898-904.

51. El-Hattab AW, Shaheen R, Hertecant J, Galadari HI, Albaqawi BS, Nabil A, et al. On the phenotypic spectrum of serine biosynthesis defects. J Inherit Metab Dis. 2016;39:373-81.

52. Alazami AM, Awad SM, Coskun S, Al-Hassan S, Hijazi H, Abdulwahab FM, et al. TLE6 mutation causes the earliest known human embryonic lethality. Genome Biol. 2015:16:1-8.

53. Maddirevula S, Awartani K, Coskun S, AlNaim LF, Ibrahim N, Abdulwahab F, et al. A genomics approach to females with infertility and recurrent pregnancy loss. Hum Genet. 2020:1-9.

54. Maddirevula S, Coskun S, Awartani K, Alsaif H, Abdulwahab F, Alkuraya F. The human knockout phenotype of PADI6 is female sterility caused by cleavage failure of their fertilized eggs. Clin Genet. 2017;91:344-5.

55. Shamseldin HE, A.L., Maddirevula S, Alsaif HS, Alzahrani F, Ewida N, Hashem M, Abdulwahab F, and Abuyousef O, K.H., Gao X, Molecular Autopsy Consortium*, Alkuraya FS. (2021). Sequencing data for a molecular autopsy study. NCBI Sequence Read Archive PRJNA761889 http://www.ncbi.nIm.nih. gov/bioproject/761889.

\section{Publisher's Note}

Springer Nature remains neutral with regard to jurisdictional claims in published maps and institutional affiliations.

Ready to submit your research? Choose BMC and benefit from:

- fast, convenient online submission

- thorough peer review by experienced researchers in your field

- rapid publication on acceptance

- support for research data, including large and complex data types

- gold Open Access which fosters wider collaboration and increased citations

- maximum visibility for your research: over $100 \mathrm{M}$ website views per year

At $\mathrm{BMC}$, research is always in progress.

Learn more biomedcentral.com/submission 